\title{
Navier-Stokes Equations with Delays
}

\author{
By Tomás Caraballo, \& José Real $\dagger$ \\ Departamento de Ecuaciones Diferenciales y Análisis Numérico, \\ Universidad de Sevilla, \\ Apdo. de Correos 1160, \\ 41080-Sevilla. \\ Spain
}

Some results on the existence and uniqueness of solutions to Navier-Stokes equations when the external force contains some hereditary characteristics are proved.

Keywords: Navier-Stokes equations, variable and distributed delays.

\section{Introduction and statement of the problem}

The Navier-Stokes equations govern the motion of usual fluids like water, air, oil, etc. These equations have been the object of numerous works since the first paper of Leray was published in 1933 (see Constantin \& Foias 1988; Lions 1969; Temam 1979, and the references therein). However, up to date, we have not found in the literature any work which takes into account the possibility of appearing some kind of delay in these equations. The main aim of this work is to consider several situations in which the external force contains some hereditary features and prove existence of solutions. These situations may appear when we want to control the system (in certain sense) by applying a force which takes into account not only the present state of the system but the history of the solution. It is worth pointing out that a similar analysis was carried out by Artola (1969) for general linear partial differential equations with delays.

Let $\Omega \subset \mathbb{R}^{N}$ ( $N=2$ or 3 ) be an open and bounded set with regular boundary $\Gamma, T>0$ given, and consider the following functional Navier-Stokes problem (for further details and notations see Lions 1969 and Temam 1979):

$$
\left\{\begin{array}{l}
\frac{\partial u}{\partial t}-\nu \Delta u+\sum_{i=1}^{N} u_{i} \frac{\partial u}{\partial x_{i}}=f-\nabla p+g\left(t, u_{t}\right) \quad \text { in }(0, T) \times \Omega, \\
\operatorname{div} u=0 \text { in }(0, T) \times \Omega, \\
u=0 \text { on }(0, T) \times \Gamma \\
u(0, x)=u_{0}(x), \quad x \in \Omega, \\
u(t, x)=\phi(t, x), t \in(-h, 0) \quad x \in \Omega
\end{array}\right.
$$

where we assume that $\nu>0$ is the kinematic viscosity, $u$ is the velocity field of the fluid, $p$ the pressure, $u_{0}$ the initial velocity field, $f$ a nondelayed external force field, $g$ another external force containing some hereditary characteristic and $\phi$ the initial datum in the interval of time $(-h, 0)$, where $h$ is a positive fixed number.

† E-mails: caraball@cica.es ; real@numer.us.es 
To start, we consider the following usual abstract spaces:

$$
\mathcal{V}=\left\{u \in\left(C_{0}^{\infty}(\Omega)\right)^{N}: \operatorname{div} u=0\right\},
$$

$H=$ the closure of $\mathcal{V}$ in $\left(L^{2}(\Omega)\right)^{N}$ with the norm $|\cdot|$, and inner product $(\cdot, \cdot)$ where for $u, v \in\left(L^{2}(\Omega)\right)^{N}$,

$$
(u, v)=\sum_{j=1}^{N} \int_{\Omega} u_{j}(x) v_{j}(x) d x,
$$

$V=$ the closure of $\mathcal{V}$ in $\left(H_{0}^{1}(\Omega)\right)^{N}$ with the norm $\|\cdot\|$, and associated scalar product $((\cdot, \cdot))$, where for $u, v \in\left(H_{0}^{1}(\Omega)\right)^{N}$,

$$
((u, v))=\sum_{i, j=1}^{N} \int_{\Omega} \frac{\partial u_{j}}{\partial x_{i}} \frac{\partial v_{j}}{\partial x_{i}} d x .
$$

It follows that $V \subset H \equiv H^{\prime} \subset V^{\prime}$, where the injections are dense and compact. Now we denote $a(u, v)=((u, v))$, and define the trilinear form $b$ on $V \times V \times V$ by

$$
b(u, v, w)=\sum_{i, j=1}^{N} \int_{\Omega} u_{i} \frac{\partial v_{j}}{\partial x_{i}} w_{j} d x \quad \forall u, v, w \in V .
$$

Let $X$ be a Banach space. Given a function $u:(-h, T) \rightarrow X$, for each $t \in(0, T)$ we denote by $u_{t}$ the function defined on $(-h, 0)$ by the relation $u_{t}(s)=u(t+s), s \in$ $(-h, 0)$.

Finally, we will use $\|\cdot\|_{*}$ for the norm in $V^{\prime}$ and $\langle\cdot, \cdot\rangle$ for the duality $\left\langle V^{\prime}, V\right\rangle$.

In order to state the problem in the correct framework, let us firstly establish the suitable assumptions on the term in which the delay is present.

In a general way, let $X$ and $Y$ be two separable Banach spaces, and $g:[0, T] \times$ $C^{0}([-h, 0] ; X) \rightarrow Y$ such that able,

(I) for all $\xi \in C^{0}([-h, 0] ; X)$, the mapping $t \in[0, T] \rightarrow g(t, \xi) \in Y$ is measur-

(II) for each $t \in[0, T], g(t, 0)=0$,

(III) there exists $L_{g}>0$ such that $\forall t \in[0, T], \quad \forall \xi, \eta \in C^{0}([-h, 0] ; X)$

$$
\|g(t, \xi)-g(t, \eta)\|_{Y} \leq L_{g}\|\xi-\eta\|_{C^{0}([-h, 0] ; X)},
$$

(IV) there exists $C_{g}>0$ such that $\forall t \in[0, T], \forall u, v \in C^{0}([-h, T] ; X)$

$$
\int_{0}^{t}\left\|g\left(s, u_{s}\right)-g\left(s, v_{s}\right)\right\|_{Y}^{2} d s \leq C_{g} \int_{-h}^{t}\|u(s)-v(s)\|_{X}^{2} d s .
$$

Observe that (I)-(III) imply that given $u \in C^{0}([-h, T] ; X)$, the function $g_{u}: t \in$ $[0, T] \rightarrow Y$ defined by $g_{u}(t)=g\left(t, u_{t}\right) \forall t \in[0, T]$, is measurable (see Bensoussan et al. 1992) and, in fact, belongs to $L^{\infty}(0, T ; Y)$. Then, thanks to (IV), the mapping

$$
\mathcal{G}: u \in C^{0}([-h, T] ; X) \rightarrow g_{u} \in L^{2}(0, T ; Y)
$$


has a unique extension to a mapping $\widetilde{\mathcal{G}}$ which is uniformly continuous from $L^{2}(-h, T ; X)$ into $L^{2}(0, T ; Y)$. From now on, we will denote $g\left(t, u_{t}\right)=\widetilde{\mathcal{G}}(u)(t)$ for each $u \in$ $L^{2}(-h, T ; X)$, and thus, $\forall t \in[0, T], \forall u, v \in L^{2}(-h, T ; X)$, we will have

$$
\int_{0}^{t}\left\|g\left(s, u_{s}\right)-g\left(s, v_{s}\right)\right\|_{Y}^{2} d s \leq C_{g} \int_{-h}^{t}\|u(s)-v(s)\|_{X}^{2} d s .
$$

With the convention above, assume that $u_{0} \in H, \phi \in L^{2}(-h, 0 ; V), f \in L^{2}\left(0, T ; V^{\prime}\right)$, $g_{1}:[0, T] \times C^{0}([-h, 0] ; V) \rightarrow\left(L^{2}(\Omega)\right)^{N}$ satisfies hypotheses (I)-(IV) with $X=V$, $Y=\left(L^{2}(\Omega)\right)^{N}, L_{g_{1}}=L_{1}$ and $C_{g_{1}}=C_{1}$, and $g_{2}:[0, T] \times C^{0}([-h, 0] ; V) \rightarrow V^{\prime}$ satisfies hypotheses (I)-(IV) with $X=V, Y=V^{\prime}, L_{g_{2}}=L_{2}$ and $C_{g_{2}}=C_{2}$.

We are interested in the following problem:

$$
\left\{\begin{array}{l}
\text { To find } u \in L^{2}(-h, T ; V) \cap L^{\infty}(0, T ; H) \text { such that, for all } v \in V, \\
\frac{d}{d t}(u(t), v)+\nu a(u(t), v)+b(u(t), u(t), v)=\langle f(t), v\rangle+\left(g_{1}\left(t, u_{t}\right), v\right) \\
\quad+\left\langle g_{2}\left(t, u_{t}\right), v\right\rangle, \\
u(0)=u_{0}, u(t)=\phi(t), \quad t \in(-h, 0), \quad
\end{array}\right.
$$

where the equation in (1.1) must be understood in the sense of $\mathcal{D}^{\prime}(0, T)$.

Remark 1.1. Observe that the terms in (1.1) are well defined. In particular, by hypotheses (I)-(IV), if $u \in L^{2}(-h, T ; V)$ the term $g_{1}\left(t, u_{t}\right)$ defines a function in $L^{2}\left(0, T ;\left(L^{2}(\Omega)^{N}\right)\right.$, and the term $g_{2}\left(t, u_{t}\right)$ defines a function in $L^{2}\left(0, T ; V^{\prime}\right)$. Thus (see Lions 1969), if $u \in L^{2}(-h, T ; V) \cap L^{\infty}(0, T ; H)$ satisfies the equation in (1.1), $u$ is weakly continuous from $[0, T]$ into $H$, and therefore the initial condition $u(0)=u_{0}$ makes sense. Of course, for $N=2$, if there exists a solution u to the problem (1.1), it then belongs to the space $C^{0}([0, T] ; H)$.

In the next section, we shall prove existence of solutions to (1.1) and the uniqueness of solution to the problem in the case $N=2$. In Section 3 , we show several general situations containing delayed terms including, in particular, those with variable and distributed delays, and we conclude the work by proving, in the Appendix, a finite-dimensional result needed for the proof of the existence of solutions to (1.1).

\section{Existence of solutions}

In this section we will prove a general theorem on the existence of solutions when $N=2$ or 3 , and uniqueness if $N=2$.

Theorem 2.1. Let us consider $u_{0} \in H, \phi \in L^{2}(-h, 0 ; V), f \in L^{2}\left(0, T ; V^{\prime}\right)$, and assume that $g_{1}:[0, T] \times C^{0}([-h, 0] ; V) \rightarrow\left(L^{2}(\Omega)\right)^{N}$ satisfies hypotheses (I)(IV) with $X=V, Y=\left(L^{2}(\Omega)\right)^{N}, L_{g_{1}}=L_{1}$ and $C_{g_{1}}=C_{1}$, and $g_{2}:[0, T] \times$ $C^{0}([-h, 0] ; V) \rightarrow V^{\prime}$ satisfies hypotheses (I)-(IV) with $X=V, Y=V^{\prime}, L_{g_{2}}=L_{2}$ and $C_{g_{2}}=C_{2}$. Then:

a) If $N=2$ and $\nu^{2}>C_{2}$, there exists at most one solution to problem (1.1).

b) If $N \in\{2,3\}$ and $\nu^{2}>C_{2}$, there exists a solution to (1.1) if, in addition, the following assumption (C) holds:

(C) If $v^{m}$ converges weakly to $v$ in $L^{2}(-h, T ; V)$ and strongly in $L^{2}(-h, T ; H)$, then $g_{i}\left(\cdot, v^{m}\right)$ converges weakly to $g_{i}(\cdot, v$. $)$ in $L^{2}\left(0, T ; V^{\prime}\right)$ for $i=1,2$. 
Proof. a) If $N=2$ and $\nu^{2}>C_{2}$, let $u, v$ be two solutions to (1) and set $w=u-v$. Then, from the energy equality, and the bounds for the trilinear form (see Lions 1969), it follows that for all $t \in(0, T)$

$$
\begin{aligned}
|w(t)|^{2}+2 \nu \int_{0}^{t}\|w(s)\|^{2} d s= & -2 \int_{0}^{t} b(w(s), u(s), w(s)) d s \\
& +2 \int_{0}^{t}\left(g_{1}\left(s, u_{s}\right)-g_{1}\left(s, v_{s}\right), w(s)\right) d s \\
& +2 \int_{0}^{t}\left\langle g_{2}\left(s, u_{s}\right)-g_{2}\left(s, v_{s}\right), w(s)\right\rangle d s \\
\leq \quad & 2 k_{1} \int_{0}^{t}|w(s)|(\|w(s)\|)\|u(s)\| d s \\
& +2 \int_{0}^{t}\left|g_{1}\left(s, u_{s}\right)-g_{1}\left(s, v_{s}\right)\right||w(s)| d s \\
& +2 \int_{0}^{t}\left\|g_{2}\left(s, u_{s}\right)-g_{2}\left(s, v_{s}\right)\right\|_{*}\|w(s)\| d s .
\end{aligned}
$$

Then, from assumption (IV), taking into account that $w(s)=0$ for $s \in(-h, 0)$, and denoting $2 \varepsilon=\nu-\sqrt{C_{2}}>0$, we have for all $t \in(0, T)$

$$
\begin{aligned}
|w(t)|^{2}+2 \nu \int_{0}^{t}\|w(s)\|^{2} d s \leq & \frac{k_{1}^{2}}{\varepsilon} \int_{0}^{t}|w(s)|^{2}\|v(s)\|^{2} d s+\varepsilon \int_{0}^{t}\|w(s)\|^{2} d s \\
& +\frac{C_{1}}{\varepsilon} \int_{0}^{t}|w(s)|^{2} d s+\varepsilon \int_{0}^{t}\|w(s)\|^{2} d s \\
& +2 \sqrt{C_{2}} \int_{0}^{t}\|w(s)\|^{2} d s
\end{aligned}
$$

and so,

$$
|w(t)|^{2}+2 \varepsilon \int_{0}^{t}\|w(s)\|^{2} d s \leq \frac{k_{1}^{2}}{\varepsilon} \int_{0}^{t}|w(s)|^{2}\|v(s)\|^{2} d s+\frac{C_{1}}{\varepsilon} \int_{0}^{t}|w(s)|^{2} d s,
$$

from which uniqueness follows thanks to the Gronwall lemma.

b) Now, we assume $N \in\{2,3\}, \nu^{2}>C_{2}$ and that condition (C) holds. For the proof of existence, we will follow a Galerkin scheme similar to the one in Constantin \& Foias (1988), so we only emphasize the details involving the new terms $g_{i}$.

Let us consider $\left\{w_{j}\right\} \subset V \cap\left(H^{2}(\Omega)\right)^{N}$ the orthonormal basis of $H$ of all the eigenfunctions of the Stokes problem in $\Omega$ with homogeneous Dirichlet conditions. The subspace of $V$ spanned by $w_{1}, \ldots, w_{m}$ will be denoted $V_{m}$. Consider the projector $P_{m}: H \rightarrow V_{m}$ given by $P_{m} u=\sum_{j=1}^{m}\left(u, w_{j}\right) w_{j}$, and define $u^{m}(t)=\sum_{j=1}^{m} \gamma_{m j}(t) w_{j}$, where

$$
\left\{\begin{array}{l}
u^{m} \in L^{2}\left(-h, T ; V_{m}\right) \cap C^{0}\left([0, T] ; V_{m}\right) \\
\frac{d}{d t}\left(u^{m}(t), w_{j}\right)+\nu a\left(u^{m}(t), w_{j}\right)+b\left(u^{m}(t), u^{m}(t), w_{j}\right)=\left\langle f(t), w_{j}\right\rangle+ \\
\quad+\left(g_{1}\left(t, u_{t}^{m}\right), w_{j}\right)+\left\langle g_{2}\left(t, u_{t}^{m}\right), w_{j}\right\rangle \quad \text { in } \mathcal{D}^{\prime}(0, T), \quad 1 \leq j \leq m, \\
u^{m}(0)=P_{m} u_{0}, u^{m}(t)=P_{m} \phi(t), \quad t \in(-h, 0) .
\end{array}\right.
$$

The preceding is a system of ordinary functional differential equations in the unknown $\gamma^{m}(t)=\left(\gamma_{m 1}(t), \ldots, \gamma_{m m}(t)\right)$. We can get existence and uniqueness of solution by applying Theorem 3.1 in the Appendix. Observe that, according to Theorem 3.1, we can ensure that problem (2.1) has one solution defined in an interval $\left[0, t_{*}\right]$ with $0<t_{*} \leq T$. However, as can be deduced by the a priori estimates below, we can set $t_{*}=T$. 
In fact, multiplying in (2.1) by $\gamma_{m j}(t)$ and summing in $j$, we get for all $t \in\left[0, t_{*}\right]$

$$
\begin{aligned}
\left|u^{m}(t)\right|^{2}+2 \nu \int_{0}^{t}\left\|u^{m}(s)\right\|^{2} d s \leq & \left|u_{0}\right|^{2}+2 \int_{0}^{t}\left\langle f(s), u^{m}(s)\right\rangle \\
& +2 \int_{0}^{t}\left(g_{1}\left(s, u_{s}^{m}\right), u^{m}(s)\right) d s \\
& +2 \int_{0}^{t}\left\langle g_{2}\left(s, u_{s}^{m}\right), u^{m}(s)\right\rangle d s,
\end{aligned}
$$

and arguing in a similar manner as we did in the proof of uniqueness in the 2dimensional case, we easily get two constants (depending on $\phi, \nu, f, g_{1}, g_{2}, h, T$, but not on $m$ nor $t_{*}$ ) $K_{1}$ and $K_{2}$ such that

$$
\sup _{t \in\left[0, t_{*}\right]}\left|u^{m}(t)\right|^{2} \leq K_{1}, \quad \int_{0}^{t_{*}}\left\|u^{m}(s)\right\|^{2} d s \leq K_{2} .
$$

So we can take $t_{*}=T$, and obtain that $\left\{u^{m}\right\}$ is bounded in $L^{2}(0, T ; V) \cap L^{\infty}(0, T ; H)$. Moreover, observe that $u^{m}=P_{m} \phi$ in $(-h, 0)$ and, by the choice of the basis $\left\{w_{j}\right\}$, the sequence $u^{m}$ converges to $\phi$ in $L^{2}(-h, 0 ; V)$, and, in particular, $g_{1}\left(\cdot, u_{.}^{m}\right)+$ $g_{2}\left(\cdot, u^{m}\right)$ is bounded in $L^{2}\left(0, T ; V^{\prime}\right)$. Now, it is a standard matter to bound the nonlinear term $b\left(u^{m}, u^{m}, \cdot\right)$, and using the same reasoning that in Constantin \& Foias (1988) (see page 67), one can obtain that $\left\{\frac{d u^{m}}{d t}\right\}$ is bounded in $L^{4 / 3}\left(0, T ; V^{\prime}\right)$ (in fact, if $N=2,\left\{\frac{d u^{m}}{d t}\right\}$ is bounded in $L^{2}\left(0, T ; V^{\prime}\right)$ ). Using the compactness of the injection of the space $W=\left\{u \in L^{2}(0, T ; V): \frac{d u}{d t} \in L^{4 / 3}\left(0, T ; V^{\prime}\right)\right\}$ into $L^{2}(0, T ; H)$, from the preceding analysis and the assumptions on $g_{1}$ and $g_{2}$, we can deduce that there exist a subsequence (denoted again $u^{m}$ ) and $u \in L^{2}(-h, T ; V)$ such that:

$$
\begin{aligned}
& u^{m} \rightarrow u \text { weakly in } L^{2}(-h, T ; V), \\
& u^{m} \rightarrow u \text { weakly star in } L^{\infty}(0, T ; H), \\
& u^{m} \rightarrow u \text { in } L^{2}(-h, T ; H), \\
& g_{i}\left(\cdot, u^{m}\right) \rightarrow g_{i}(\cdot, u .) \text { weakly in } L^{2}\left(0, T ; V^{\prime}\right), \quad i=1,2 .
\end{aligned}
$$

Now, as in the non-delay case, we can take limits in (2.1) after integrating over the interval $(0, t)$ (for $t \in(0, T)$ ), getting that $u$ is a solution to our problem (1.1) (see once again, e.g., Constantin \& Foias 1988 for the complete details).

Remark 2.2. Observe that if $g_{1}$ satisfies (I)-(IV) with $X=H$ and $Y=\left(L^{2}(\Omega)\right)^{N}$, then, as a direct consequence of (IV), $g_{1}$ satisfies assumption $(C)$.

\section{Some general situations}

In this section, we are going to show some situations where our theory can be applied. The cases considered include situations such as distributed delay, variable delay, and delay in gradient or second order derivatives terms.

(a) Case 1

Let $G:[0, T] \times \mathbb{R}^{N} \rightarrow \mathbb{R}^{N}$ be a measurable function satisfying $G(t, 0)=0$ for all $t \in[0, T]$, and assume that there exists $L_{1}>0$ such that

$$
|G(t, u)-G(t, v)|_{\mathbb{R}^{N}} \leq L_{1}|u-v|_{\mathbb{R}^{N}}, \forall u, v \in \mathbb{R}^{N} .
$$


Consider a function $\omega(t)$, which is going to play the role of the delay function. We suppose that $\omega \in C^{1}([0, T]), \omega(t) \geq 0$ for all $t \in[0, T], h=\max _{t \in[0, T]} \omega(t)>0$ and $\omega_{*}=\max _{t \in[0, T]} \omega^{\prime}(t)<1$. Then, we define $g_{1}(t, \xi)(x)=G(t, \xi(-\omega(t))(x))$ for each $\xi \in C^{0}([0, T] ; H), x \in \Omega$ and $t \in[0, T]$. Notice that, in this case, the delayed term $g_{1}$ in our problem turns to $g_{1}\left(t, u_{t}\right)=G(t, u(t-\omega(t)))$. Then, $g_{1}$ satisfies the hypotheses in Theorem 2.1 with $X=H$ and $Y=L^{2}(\Omega)^{N}$.

Indeed, (I)-(III) follow immediately. On the other hand, if $u, v \in L^{2}(-h, T ; H)$, using the change of variable $\tau=s-\omega(s)$ it is easy to see that

$$
\int_{0}^{t}\left|g_{1}\left(s, u_{s}\right)-g_{1}\left(s, v_{s}\right)\right|^{2} d s \leq \int_{-h}^{t}|u(\tau)-v(\tau)|^{2} d \tau \quad \forall t \in[0, T]
$$

and, consequently, (IV) and (C) are fulfilled.

(b) Case 2

Let now $G:[0, T] \times[-h, 0] \times \mathbb{R}^{N} \rightarrow \mathbb{R}^{N}$ be a measurable function satisfying $G(t, s, 0)=0$ for all $(t, s) \in[0, T] \times[-h, 0]$ and such that there exists a function $\gamma \in L^{2}(-h, 0)$ such that

$$
|G(t, s, u)-G(t, s, v)|_{\mathbb{R}^{N}} \leq \gamma(s)|u-v|_{\mathbb{R}^{N}}, \forall u, v \in \mathbb{R}^{N} \quad \forall(t, s) \in[0, T] \times[-h, 0] .
$$

Then, we define $g_{1}(t, \xi)(x)=\int_{-h}^{0} G(t, s, \xi(s)(x)) d s$ for each $\xi \in C^{0}([0, T] ; H), x \in \Omega$ and $t \in[0, T]$. In this case, the delayed term $g_{1}$ in our problem becomes

$$
g_{1}\left(t, u_{t}\right)=\int_{-h}^{0} G(t, s, u(t+s)) d s .
$$

As in Case 1, $g_{1}$ satisfies the hypotheses in Theorem 2.1 with $X=H$ and $Y=$ $\left(L^{2}(\Omega)\right)^{N}$.

Indeed, (I) and (II) can be deduced immediately. On the other hand, if $\xi, \eta \in$ $C^{0}([0, T] ; H)$, for each $t \in[0, T]$ we obtain

$$
\begin{aligned}
\left|g_{1}(t, \xi)-g_{1}(t, \eta)\right|^{2} & \leq \int_{\Omega}\left(\int_{-h}^{0}|G(t, s, \xi(s)(x))-G(t, s, \eta(s)(x))|_{\mathbb{R}^{N}} d s\right)^{2} d x \\
& \leq \int_{\Omega}\left(\int_{-h}^{0} \gamma(s)|\xi(s)(x)-\eta(s)(x)|_{\mathbb{R}^{N}} d s\right)^{2} d x \\
& \leq \int_{\Omega}\|\gamma\|_{L^{2}(-h, 0)}^{2}\left(\int_{-h}^{0}|\xi(s)(x)-\eta(s)(x)|_{\mathbb{R}^{N}}^{2} d s\right) d x \\
& \leq h\|\gamma\|_{L^{2}(-h, 0)}^{2}\|\xi-\eta\|_{C^{0}([0, T] ; H)}^{2} .
\end{aligned}
$$

Finally, if $u, v \in L^{2}(-h, T ; H)$ then, for each $t \in[0, T]$ it follows

$$
\int_{0}^{t}\left|g_{1}\left(\tau, u_{\tau}\right)-g_{1}\left(\tau, v_{\tau}\right)\right|^{2} d \tau \leq h\|\gamma\|_{L^{2}(-h, 0)}^{2} \int_{0}^{t}\left(\int_{-h}^{0}|u(s+\tau)-v(s+\tau)|^{2} d s\right) d \tau,
$$

and, with the change $r=s+\tau$,

$$
\begin{aligned}
\int_{0}^{t}\left|g_{1}\left(\tau, u_{\tau}\right)-g_{1}\left(\tau, v_{\tau}\right)\right|^{2} d \tau & \leq h\|\gamma\|_{L^{2}(-h, 0)}^{2} \int_{0}^{t}\left(\int_{\tau-h}^{\tau}|u(r)-v(r)|^{2} d r\right) d \tau \\
& \leq h T\|\gamma\|_{L^{2}(-h, 0)}^{2} \int_{-h}^{t}|u(r)-v(r)|^{2} d r
\end{aligned}
$$

Article submitted to Royal Society 
(c) Case 3

Now, we shall exhibit a situation where certain delay can appear in terms containing partial derivatives with respect to the spatial variables.

Let $B(\cdot) \in L^{\infty}\left(0, T ; \mathcal{L}\left(V ;\left(L^{2}(\Omega)\right)^{N}\right)\right)$ and $\omega \in C^{1}([0, T])$, such that $\omega(t) \geq 0$ for all $t \in[0, T], h=\max _{t \in[0, T]} \omega(t)>0$ and $\omega_{*}=\max _{t \in[0, T]} \omega^{\prime}(t)<1$. We now define $g_{1}(t, \xi)=B(t) \xi(-\omega(t))$ for each $\xi \in C^{0}([0, T] ; V)$, and $t \in[0, T]$. Thus, in this case the delayed term $g_{1}$ in problem (1.1) turns to $g_{1}\left(t, u_{t}\right)=B(t) u(t-\omega(t))$. It is easy to see that $g_{1}$ satisfies the hypotheses in Theorem 2.1 with $X=V$ and $Y=\left(L^{2}(\Omega)\right)^{N}$.

Indeed, (I)-(IV) obviously hold. On the other hand, if $v^{m}$ converges to zero weakly in $L^{2}(-h, T ; V)$ and $\psi \in L^{2}(0, T ; V)$ is given, we have

$$
\int_{0}^{T}\left\langle g_{1}\left(t, v_{t}^{m}\right), \psi(t)\right\rangle d t=\int_{0}^{T}\left\langle B^{*}(t) \psi(t), v^{m}(t-\omega(t))\right\rangle d t
$$

with $B^{*}(\cdot) \in L^{\infty}\left(0, T ; \mathcal{L}\left(L^{2}(\Omega)^{N} ; V^{\prime}\right)\right) \subset L^{\infty}\left(0, T ; \mathcal{L}\left(V ; V^{\prime}\right)\right)$ the adjoint of $B(\cdot)$. Using the change of variables $\tau=t-\omega(t)=\rho(t)$, we obtain

$$
\begin{aligned}
\int_{0}^{T}\left\langle g_{1}\left(t, v_{t}^{m}\right), \psi(t)\right\rangle d t & =\int_{\rho(0)}^{\rho(T)}\left\langle B^{*}\left(\rho^{-1}(\tau)\right) \psi\left(\rho^{-1}(\tau)\right), v^{m}(\tau)\right\rangle \frac{1}{\rho^{\prime}\left(\rho^{-1}(\tau)\right)} d \tau \\
& =\int_{-h}^{T}\left\langle\Psi(\tau), v^{m}(\tau)\right\rangle d \tau
\end{aligned}
$$

with

$$
\Psi(\tau)= \begin{cases}\frac{1}{\rho^{\prime}\left(\rho^{-1}(\tau)\right)} B^{*}\left(\rho^{-1}(\tau)\right) \psi\left(\rho^{-1}(\tau)\right) & \text { if } \tau \in[\rho(0), \rho(T)] \\ 0 & \text { if } \tau \in[-h, T] \backslash[\rho(0), \rho(T)]\end{cases}
$$

For this function $\Psi$ it follows

$$
\int_{-h}^{T}\|\Psi(\tau)\|_{*}^{2} d \tau=\int_{\rho(0)}^{\rho(T)} \frac{1}{\left(\rho^{\prime}\left(\rho^{-1}(\tau)\right)\right)^{2}}\left\|B^{*}\left(\rho^{-1}(\tau)\right) \psi\left(\rho^{-1}(\tau)\right)\right\|_{*}^{2} d \tau
$$

and thus, by means of the change $\tau=\rho(t)=t-\omega(t)$,

$$
\int_{-h}^{T}\|\Psi(\tau)\|_{*}^{2} d \tau=\int_{0}^{T} \frac{1}{1-\omega^{\prime}(t)}\left\|B^{*}(t) \psi(t)\right\|_{*}^{2} d t \leq \frac{b_{0}^{2}}{1-\omega_{*}} \int_{0}^{T}\|\psi(t)\|^{2} d t
$$

where $b_{0}=\left\|B^{*}(\cdot)\right\|_{L^{\infty}\left(0, T ; \mathcal{L}\left(V ; V^{\prime}\right)\right)}$. Consequently, $\Psi \in L^{2}\left(-h, T ; V^{\prime}\right)$ and

$$
\lim _{m \rightarrow \infty} \int_{0}^{T}\left\langle g_{1}\left(t, v_{t}^{m}\right), \psi(t)\right\rangle d t=\lim _{m \rightarrow \infty} \int_{-h}^{T}\left\langle\Psi(\tau), v^{m}(\tau)\right\rangle d \tau=0
$$

Therefore, hypothesis (C) is satisfied and once again we can apply our theory to this situation. 
(d) Case 4

Let $K \in L^{\infty}\left(-h, T ; \mathcal{L}\left(V ; V^{\prime}\right)\right)$ and consider in problem (1.1) a term of the form $g_{2}\left(t, u_{t}\right)=\int_{-h}^{0} K(t+s) u(t+s) d s$, defined for all $u \in L^{2}(-h, T ; V)$. This term corresponds to the situation $g_{2}(t, \xi)=\int_{-h}^{0} K(t+s) \xi(s) d s$ for each $t \in[0, T]$ and $\xi \in$ $C^{0}([0, T] ; V)$. In this case, it is easy to see that $g_{2}$ is well defined and satisfies (I)-(IV) with $X=V$ and $Y=V^{\prime}$. In particular, if we denote $k=\|K(\cdot)\|_{L^{\infty}\left(-h, T ; \mathcal{L}\left(V ; V^{\prime}\right)\right)}$, we can see that, for each $t \in[0, T]$ and each $u \in L^{2}(-h, T ; V)$, we have

$$
\int_{0}^{t}\left\|g_{2}\left(s, u_{s}\right)\right\|_{*}^{2} d s \leq k^{2} h \min (h, T) \int_{-h}^{t}\|u(s)\|^{2} d s,
$$

and thus, (IV) holds by setting $C_{2}=k^{2} h \min (h, T)$.

On the other hand, let $v^{m}$ be weakly converging to zero in $L^{2}(-h, T ; V)$, and fix $\psi \in L^{2}(0, T ; V)$. Then

$$
\int_{0}^{T}\left\langle g_{2}\left(t, v_{t}^{m}\right), \psi(t)\right\rangle d t=\int_{0}^{T}\left\langle\int_{t-h}^{t} K(\tau) v^{m}(\tau) d \tau, \psi(t)\right\rangle d t
$$

and, by Fubini's theorem, it is easy to see that

$$
\int_{0}^{T}\left\langle g_{2}\left(t, v_{t}^{m}\right), \psi(t)\right\rangle d t=\int_{-h}^{T}\left\langle\Sigma(\tau), v^{m}(\tau)\right\rangle d \tau
$$

with $\Sigma(\tau)=K^{*}(\tau) \Psi(\tau)$ and

$$
\Psi(\tau)= \begin{cases}\int_{0}^{\tau+h} \psi(t) d t & \text { if }-h \leq \tau<0 \\ \int_{\tau}^{\tau+h} \psi(t) d t & \text { if } 0 \leq \tau<T-h \\ \int_{\tau}^{T} \psi(t) d t & \text { if } T-h \leq \tau \leq T\end{cases}
$$

in the case $h \leq T$, and

$$
\Psi(\tau)= \begin{cases}\int_{0}^{\tau+h} \psi(t) d t & \text { if }-h \leq \tau<T-h, \\ \int_{0}^{T} \psi(t) d t & \text { if } T-h \leq \tau<0, \\ \int_{\tau}^{T} \psi(t) d t & \text { if } 0 \leq \tau \leq T,\end{cases}
$$

in the case $h>T$. In both cases $\Psi \in C^{0}([0, T] ; V)$, and in particular $\Sigma \in L^{2}\left(0, T ; V^{\prime}\right)$. Consequently, if $v^{m}$ converges weakly to zero in $L^{2}(-h, T ; V)$, then $g_{2}\left(\cdot, v^{m}\right)$ converges weakly to zero in $L^{2}\left(-h, T ; V^{\prime}\right)$ and thus, $g_{2}$ satisfies hypothesis $(C)$.

\section{Appendix}

In this section we will prove a theorem on the existence of solutions for a finitedimensional problem. This result has been used in the proof of Theorem 2.1, and is a variant of Theorem 3.2 Chapter 4 (p. 213) in Bensoussan et al. (1992). We present the proof for the sake of completeness. However, it is worth mentioning that a similar result is proved in Hale \& Lunel (1995) in the case of continuous initial datum, although this result cannot be applied to our situation. 
Theorem 3.1. Let $u_{0} \in \mathbb{R}^{m}, \phi \in L^{2}\left(-h, 0 ; \mathbb{R}^{m}\right), k \in L^{2}\left(0, T ; \mathbb{R}^{m}\right), g:[0, T] \times$ $C^{0}\left([-h, 0] ; \mathbb{R}^{m}\right) \rightarrow \mathbb{R}^{m}$ satisfying hypotheses (I)-(IV) with $X=Y=\mathbb{R}^{m}$, and $f:[0, T] \times \mathbb{R}^{m} \rightarrow \mathbb{R}^{m}$ a continuous function such that $f(t, 0)=0$ and for all $n>0$ there exists $L_{n}>0$ such that

$$
|f(t, u)-f(t, v)|_{\mathbb{R}^{m}} \leq L_{n}|u-v|_{\mathbb{R}^{m}}, \quad \forall|u|_{\mathbb{R}^{m}} \leq n,|v|_{\mathbb{R}^{m}} \leq n, \quad \forall t \in[0, T] .
$$

Then:

a) For each $t_{*} \in(0, T]$ there exists at most one solution to the problem

$$
\left\{\begin{array}{l}
\text { To find } u \in L^{2}\left(-h, t_{*} ; \mathbb{R}^{m}\right) \cap C^{0}\left(\left[0, t_{*}\right] ; \mathbb{R}^{m}\right) \text { such that } \\
u(t)=\phi(t), \quad t \in(-h, 0), \\
u(t)=u_{0}+\int_{0}^{t} f(s, u(s)) d s+\int_{0}^{t} g\left(s, u_{s}\right) d s+\int_{0}^{t} k(s) d s \quad \forall t \in\left[0, t_{*}\right] .
\end{array}\right.
$$

b) There exists $t_{*} \in(0, T]$ such that there exists one (and only one) solution to the problem (3.1).

c) Suppose that there exists a constant $C>0$ such that if $t_{*} \in(0, T]$ is such that there is a solution $u$ of (3.1), then $\max _{t \in\left[0, t_{*}\right]}|u(t)|_{\mathbb{R}^{m}} \leq C$. Then, under this additional assumption, there exists a solution to problem (3) with $t_{*}=T$.

Proof. a) If $u$ and $v$ are two solutions of (3.1) then, denoting $w=u-v$, we obtain $w=0$ in $(-h, 0)$, and for all $t \in\left[0, t_{*}\right]$

$$
|w(t)|_{\mathbb{R}^{m}} \leq L_{n} \int_{0}^{t}|w(s)|_{\mathbb{R}^{m}} d s+\left(C_{g} t_{*} \int_{0}^{t}|w(s)|_{\mathbb{R}^{m}}^{2} d s\right)^{1 / 2}
$$

with $n=\max \left(\max _{t \in\left[0, t_{*}\right]}|u(t)|_{\mathbb{R}^{m}}, \max _{t \in\left[0, t_{*}\right]}|v(t)|_{\mathbb{R}^{m}}\right)$.

Consequently,

$$
|w(t)|_{\mathbb{R}^{m}}^{2} \leq\left(L_{n}+C_{g}^{1 / 2}\right)^{2} t_{*} \int_{0}^{t}|w(s)|_{\mathbb{R}^{m}}^{2} d s, \quad \forall t \in\left[0, t_{*}\right],
$$

and thus, $w=0$ on $\left[0, t_{*}\right]$.

b) Take any $C>0$ such that $\left|u_{0}\right|_{\mathbb{R}^{m}} \leq C$. Denote

$$
M=1+C+C_{g}^{1 / 2}\|\phi\|_{L^{2}\left(-h, 0 ; \mathbb{R}^{m}\right)}+C\left(C_{g} h\right)^{1 / 2},
$$

and fix $t_{*} \in(0, T]$ such that

$$
2 t_{*} \leq\left(1+M\left(L_{M}+C_{g}^{1 / 2}\right)+\|k\|_{L^{2}\left(0, T ; \mathbb{R}^{m}\right)}\right)^{-2},
$$

and $\frac{T}{t_{*}}$ being an integer.

Let

$X=\left\{u \in L^{2}\left(-h, t_{*} ; \mathbb{R}^{m}\right) \cap C^{0}\left(\left[0, t_{*}\right] ; \mathbb{R}^{m}\right) ; \quad u=\phi\right.$ in $\left.(-h, 0),|u(t)|_{\mathbb{R}^{m}} \leq M \forall t \in\left[0, t_{*}\right]\right\}$,

with the metric $d(\cdot, \cdot)$ given by

$$
d(u, v)=\max _{t \in\left[0, t_{*}\right]}|u(t)-v(t)|_{\mathbb{R}^{m}}
$$

Article submitted to Royal Society 
It is evident that $(X, d)$ is a complete metric space and $X \neq \emptyset$.

For each $u \in X$, denote

$$
\mathcal{T} u(t)= \begin{cases}\phi(t), & \text { if } t \in(-h, 0), \\ u_{0}+\int_{0}^{t} f(s, u(s)) d s+\int_{0}^{t} g\left(s, u_{s}\right) d s+\int_{0}^{t} k(s) d s, & \text { if } t \in\left[0, t_{*}\right] .\end{cases}
$$

It is obvious that $\mathcal{T} u \in L^{2}\left(-h, t_{*} ; \mathbb{R}^{m}\right) \cap C^{0}\left(\left[0, t_{*}\right] ; \mathbb{R}^{m}\right)$ and, by definition, $\mathcal{T} u(t)=$ $\phi(t)$ if $t \in(-h, 0)$. Moreover, if $t \in\left[0, t_{*}\right]$,

$|\mathcal{T} u(t)|_{\mathbb{R}^{m}} \leq\left|u_{0}\right|_{\mathbb{R}^{m}}+L_{M} M t_{*}+\left(C_{g} t_{*}\right)^{1 / 2}\left(\int_{-h}^{t}|u(s)|_{\mathbb{R}^{m}}^{2}\right)^{1 / 2}+\left(t_{*}\right)^{1 / 2}\|k\|_{L^{2}\left(0, T ; \mathbb{R}^{m}\right)}$,

and consequently, thanks to the choice of $t_{*},|\mathcal{T} u(t)|_{\mathbb{R}^{m}} \leq M$ for all $t \in\left[0, t_{*}\right]$. Thus, $\mathcal{T}$ is a mapping from $X$ into $X$. Finally, it is not difficult to see that $\sqrt{2} d(\mathcal{T} u, \mathcal{T} v) \leq$ $d(u, v)$ for all $u, v \in X$. Consequently, $\mathcal{T}$ has a fixed point in $X$.

c) If there exists a constant $C>0$ such that if $t_{*} \in(0, T]$ is such that there is a solution $u$ of $(3.1)$, then $\max _{t \in\left[0, t_{*}\right]}|u(t)|_{\mathbb{R}^{m}} \leq C$, with this $C$ fixed, take $M$ and $t_{*}$ as in b), that is,

$$
M=1+C+C_{g}^{1 / 2}\|\phi\|_{L^{2}\left(-h, 0 ; \mathbb{R}^{m}\right)}+C\left(C_{g} h\right)^{1 / 2}
$$

and $t_{*} \in(0, T]$ such that

$$
2 t_{*} \leq\left(1+M\left(L_{M}+C_{g}^{1 / 2}\right)+\|k\|_{L^{2}\left(0, T ; \mathbb{R}^{m}\right)}\right)^{-2}
$$

with $\frac{T}{t_{*}}$ being an integer.

We know that then, there exists one and only one function $u \in L^{2}\left(-h, t_{*} ; \mathbb{R}^{m}\right) \cap$ $C^{0}\left(\left[0, t_{*}\right] ; \mathbb{R}^{m}\right)$ such that

$$
\left\{\begin{array}{l}
u(t)=\phi(t), \quad t \in(-h, 0) \\
u(t)=u_{0}+\int_{0}^{t} f(s, u(s)) d s+\int_{0}^{t} g\left(s, u_{s}\right) d s+\int_{0}^{t} k(s) d s, \quad \forall t \in\left[0, t_{*}\right]
\end{array}\right.
$$

If $t_{*}<T$, then denote $\widetilde{\phi}(s)=u\left(t_{*}+s\right)$ for each $s \in(-h, 0)$, and consider the problem

$$
\left\{\begin{aligned}
v \in L^{2}\left(-h, t_{*} ; \mathbb{R}^{m}\right) \cap C^{0}\left(\left[0, t_{*}\right] ; \mathbb{R}^{m}\right) \\
v(t)=\widetilde{\phi}(t), \quad t \in(-h, 0) \\
v(t)=u\left(t_{*}\right)+\int_{0}^{t} f\left(s+t_{*}, v(s)\right) d s+\int_{0}^{t} g\left(s+t_{*}, v_{s}\right) d s \\
\quad+\int_{0}^{t} k\left(s+t_{*}\right) d s, \quad \forall t \in\left[0, t_{*}\right] .
\end{aligned}\right.
$$

The problem (3.2) has the same structure than (3.1), with $\left|u\left(t_{*}\right)\right|_{\mathbb{R}^{m}} \leq C$ and

$$
\int_{-h}^{0}|\widetilde{\phi}(s)|_{\mathbb{R}^{m}}^{2} d s=\int_{t_{*}-h}^{t_{*}}|u(\tau)|_{\mathbb{R}^{m}}^{2} d \tau \leq C^{2} h+\|\phi\|_{L^{2}\left(-h, 0 ; \mathbb{R}^{m}\right)}^{2} .
$$

Moreover, if we denote

$$
\tilde{f}(t, w)=f\left(t+t_{*}, w\right) \quad \forall t \in\left[0, t_{*}\right] \quad \forall w \in \mathbb{R}^{m},
$$


and

$$
\widetilde{g}(t, \xi)=f\left(t+t_{*}, \xi\right) \quad \forall t \in\left[0, t_{*}\right] \quad \forall \xi \in C^{0}\left([-h, 0] ; \mathbb{R}^{m}\right)
$$

then it is easy to see that $\widetilde{f}$ and $\widetilde{g}$ satify the hypotheses in the theorem, with $T$ substituted by $t_{*}$, and the same constants $L_{n}, C_{g}$ and $L_{g}$.

For example, if $u, v \in C^{0}\left(\left[-h, t_{*}\right] ; \mathbb{R}^{m}\right)$ and $t \in\left[0, t_{*}\right]$, then

$$
\begin{aligned}
\int_{0}^{t}\left|\widetilde{g}\left(s, u_{s}\right)-\widetilde{g}\left(s, v_{s}\right)\right|_{\mathbb{R}^{m}}^{2} d s & =\int_{0}^{t}\left|g\left(s+t_{*}, u_{s}\right)-g\left(s+t_{*}, v_{s}\right)\right|_{\mathbb{R}^{m}}^{2} d s \\
& =\int_{t_{*}}^{t_{*}+t}\left|g\left(\tau, u_{\tau-t_{*}}\right)-g\left(\tau, v_{\tau-t_{*}}\right)\right|_{\mathbb{R}^{m}}^{2} d \tau .
\end{aligned}
$$

If we denote

$$
\widehat{u}(s)=\left\{\begin{array}{ll}
u\left(s-t_{*}\right) & \text { if } s \in\left[t_{*}-h, 2 t_{*}\right], \\
0 & \text { if } s \in\left[-h, t_{*}-h\right),
\end{array} \widehat{v}(s)= \begin{cases}v\left(s-t_{*}\right) & \text { if } s \in\left[t_{*}-h, 2 t_{*}\right], \\
0 & \text { if } s \in\left[-h, t_{*}-h\right),\end{cases}\right.
$$

then $\widehat{u}$ and $\widehat{v}$ are in $L^{2}\left(-h, 2 t_{*} ; \mathbb{R}^{m}\right)$, and $\forall \tau \in\left[t_{*}, t_{*}+t\right], \forall \theta \in[-h, 0]$,

$$
u_{\tau-t_{*}}(\theta)=\widehat{u}_{\tau}(\theta), \quad v_{\tau-t_{*}}(\theta)=\widehat{v}_{\tau}(\theta),
$$

thus we obtain

$$
\begin{aligned}
\int_{0}^{t}\left|\widetilde{g}\left(s, u_{s}\right)-\widetilde{g}\left(s, v_{s}\right)\right|_{\mathbb{R}^{m}}^{2} d s & =\int_{t_{*}}^{t_{*}+t}\left|g\left(\tau, \widehat{u}_{\tau}\right)-g\left(\tau, \widehat{v}_{\tau}\right)\right|_{\mathbb{R}^{m}}^{2} d \tau \\
& \leq \int_{0}^{t_{*}+t}\left|g\left(\tau, \widehat{u}_{\tau}\right)-g\left(\tau, \widehat{v}_{\tau}\right)\right|_{\mathbb{R}^{m}}^{2} d \tau \\
& \leq C_{g} \int_{-h}^{t_{*}+t}|\widehat{u}(\tau)-\widehat{v}(\tau)|_{\mathbb{R}^{m}}^{2} d \tau \\
& =C_{g} \int_{t_{*}-h}^{t_{*}+t}|\widehat{u}(\tau)-\widehat{v}(\tau)|_{\mathbb{R}^{m}}^{2} d \tau \\
& =C_{g} \int_{t_{*}-h}^{t_{*}+t}\left|u\left(\tau-t_{*}\right)-v\left(\tau-t_{*}\right)\right|_{\mathbb{R}^{m}}^{2} d \tau,
\end{aligned}
$$

and, consequently,

$$
\int_{0}^{t}\left|\widetilde{g}\left(s, u_{s}\right)-\widetilde{g}\left(s, v_{s}\right)\right|_{\mathbb{R}^{m}}^{2} d s \leq C_{g} \int_{-h}^{t}|u(s)-v(s)|_{\mathbb{R}^{m}}^{2} d s .
$$

By the considerations above, we can ensure that there exists one and only one solution to (3.2). Now, it is easy to see that the function

$$
w(t)=\left\{\begin{array}{ll}
u(t) & \text { if } t \in\left(-h, t_{*}\right] \\
v(t) & \text { if } t \in\left(t_{*}, 2 t_{*}\right.
\end{array},\right.
$$

with $u$ the solution to (3.1) in $\left(-h, t_{*}\right]$, and $v$ the solution to $(3.2)$, is solution of (3.1) in the interval $\left(-h, 2 t_{*}\right]$. If $2 t_{*}=T$, we have finished, but if $2 t_{*}<T$, we then can use a similar construction to the preceding one, and obtain a solution to (3.1) in the interval $\left(-h, 3 t_{*}\right]$. After a finite number of steps, we can construct the solution of $(3.1)$ in the interval $(-h, T]$.

Partly supported by DGICYT (Spain) Proyecto PB98-1134. 


\section{References}

Artola, M. 1969 Sur les perturbations des équations d'evolution, application a des problèmes de retard, Ann. Scient. Ec. Norm. Sup. 4 série, t. 2, 137-253.

Bensoussan, A., Da Prato, G., Delfour, M.C., \& Mitter, S.K. 1992 Representation and Control of Infinite Dimensional Systems, Vol. I, Birkhäuser, Boston-Basel-Berlin.

Constantin, P. \& Foias, C. 1988 Navier Stokes Equations, The University of Chicago Press, Chicago.

Hale, J.K. \& Verduyn Lunel, S.M. 1995 Introduction to Functional Differential Equations, Springer-Verlag, New York.

Leray, J. 1933 Etude de diverses équations intégrales non linéaires et de quelques problèmes que pose l'hydrodynamique, J. Math. Pures Appl. 12, 1-82.

Lions, J.L. 1969 Quelque méthodes de résolution des problèmes aux limites non linéaires, Dunod, Gauthier- Villars, Paris.

Temam, R. 1979 Navier-Stokes equations, Theory and Numerical Analysis, 2nd. ed., North Holland, Amsterdam. 